\title{
Localized delivery of $\beta$-NGF via injectable microrods accelerates endochondral fracture repair
}

Kevin O. Rivera',2,3,a, Darnell L. Cuylear ${ }^{1,3, a}$, Victoria Duke ${ }^{4}, K^{1}$ Kelsey Marie O’Hara4 ${ }^{4}$ Bhushan N. Kharbikar ${ }^{3}$, Alex N. Kryger ${ }^{5}$, Theodore Miclau², Chelsea S. Bahney ${ }^{1,2,3,4}$, Tejal A. Desai ${ }^{1,3,6}$

${ }^{1}$ Graduate Program in Oral and Craniofacial Sciences, School of Dentistry, University of California, San Francisco (UCSF), San Francisco, CA, USA

${ }^{2}$ Department of Orthopaedic Surgery, Orthopaedic Trauma Institute, University of California, San Francisco (UCSF), San Francisco, CA, USA

${ }^{3}$ Department of Bioengineering and Therapeutic Sciences, University of California, San Francisco (UCSF), San Francisco, CA, USA

${ }^{4}$ The Steadman Philippon Research Institute (SPRI), Vail, CO, USA

${ }^{5}$ School of Dentistry, University of California, San Francisco (UCSF), San Francisco, CA, USA

${ }^{6}$ Department of Bioengineering, University of California, Berkeley (UC Berkeley), Berkeley, CA, USA

aThese authors contributed equally

Correspondence: tejal.desai@ucsf.edu. UCSF QB3 Box 2520, $17004^{\text {th }}$ Street Room 204, San Francisco, CA 94158-2330.

Conflict of interest: The authors declare no competing interests.

\section{Abstract}

Currently, there are no biological approaches to accelerate bone fracture repair. Osteobiologics that promote endochondral ossification are an exciting alternative to surgically implanted bone grafts, however, the translation of osteobiologics remains elusive because of the need for localized and sustained delivery that is both safe and effective. In this regard, an injectable system composed of hydrogel-based microparticles designed to release osteobiologics in a controlled and localized manner is ideal in the context of bone fracture repair. Here, we describe poly (ethylene glycol) dimethacrylate (PEGDMA)-based microparticles, in the form of microrods, engineered to be loaded with beta nerve growth factor ( $\beta$-NGF) for use in a murine tibial fracture model. In-vitro studies demonstrated that protein-loading efficiency is readily altered by varying PEGDMA macromer concentration and that $\beta$-NGF loaded onto PEGDMA microrods exhibited sustained release over a period of 7 days. In-vitro bioactivity of $\beta$-NGF was confirmed using a tyrosine receptor kinase $A(T r k-A)$ expressing cell line, TF-1. Moreover, TF-1 cell proliferation significantly increased when incubated with $\beta$-NGF loaded PEGDMA microrods versus $\beta$-NGF in media. In-vivo studies show that PEGDMA microrods injected into the fracture calluses of mice remained in the callus for over 7 days. Importantly, a single injection of $\beta$ NGF-loaded PEGDMA microrods resulted in significantly improved fracture healing as indicated by significant increases in bone volume, trabecular connective density, and bone mineral density and a significant decrease in cartilage despite a remarkably lower dose $(\sim 111$ fold) than the $\beta-N G F$ in media. In conclusion, we demonstrate a novel and translational method of delivering $\beta$-NGF via injectable PEGDMA microrods to improve bone fracture repair.

\section{Key words}

Keywords (6 maximum): fracture repair, endochondral ossification, beta-nerve growth factor, drug delivery, sustained release, poly (ethylene) glycol dimethacrylate

\section{Introduction}

Bone fractures are one of the most common injuries worldwide, affecting millions of individuals, and imparting billions of dollars in healthcare cost burden annually ${ }^{1,2}$. Although bones possess the ability to fully regenerate, fractures induce significant pain and loss in quality of life throughout the time course of healing, which can take 3-6 months under normal conditions ${ }^{3}$. Delayed healing or failure to heal (non-unions) occur in 5-10 \% of cases leading to healing times often exceeding 9 months and/or requiring multiple surgeries to achieve union ${ }^{4}$. Co- 
morbidities such as age, smoking, diabetes, and obesity drastically increase the prevalence of impaired bone healing to upward of $50 \%$ of fracture patients ${ }^{5,6}$. Currently, surgical application of autologous bone grafts or adjustments to the orthopaedic hardware are the standard of care approaches to stimulate healing. Surgical intervention is associated with significant challenges, including, donor site morbidity, longer recovery time, increased costs, and risk of infection, among others ${ }^{7,8}$. There are no pharmacologic reagents approved to accelerate fracture healing or prevent non-union ${ }^{1}$. Given these limitations with surgical methods, there is an unmet clinical need to develop pharmacological approaches to improve bone regeneration without surgery.

Bone morphogenetic proteins (BMPs) are perhaps the most established osteoanabolic growth factors and have been used for regenerating bone through a direct effect on osteoblastic differentiation of osteochondral progenitors $^{9}$. Of the BMPs, BMP-2 is the only osteobiologic growth factor with FDA approval for treatment of problematic fractures ${ }^{10}$. Currently clinical application of BMP-2 in fracture repair remains limited due to its uncertain efficacy, high cost, and growing list of adverse outcomes that includes inflammatory complications, ectopic bone formation, and osteolysis ${ }^{11,12}$. The serious complications are believed to be driven by the supraphysiological doses needed to achieve therapeutic efficacy and lack of drug delivery approaches to mitigate side effects. Thus, it is imperative to explore the use of novel osteobiologics to accelerate fracture repair and to develop optimal drug delivery systems to localize the biologic's effects and mitigate severe adverse outcomes.

While therapeutic use of BMPs have focused on promoting direct bone formation (intramembranous ossification), bone fractures heal primarily through endochondral ossification (EO). EO, or indirect bone formation, occurs when an avascular, aneural cartilage intermediate converts into a vascularized and innervated bone. EO fracture repair is a dynamic process that proceeds through four overlapping steps: 1) formation of hematoma at the fracture site and activation of the proinflammatory phase, 2) formation of a fibrocartilaginous callus via chondrocytes, 3) formation of a bony callus via chondrocyte hypertrophy and mineralization, and 4) remodeling and compact bone formation ${ }^{13-16}$. Although the molecular mechanisms governing chondrogenesis and hypertrophy are well described, it remains unclear which mechanisms regulate the conversion of cartilage to bone ${ }^{17,18}$. Many molecular pathways have been identified to regulate cartilage to bone transformation including transforming growth factor $\beta$ (TGF- $\beta$ ), Sox9, Runx2, Wnt, and Indian hedgehog $(\mathrm{Ihh})^{19-30}$.

Recently, nerve growth factor (NGF) signaling via its high affinity receptor tropomyosin receptor kinase $A$ (TrkA) has been shown to be important in both intramembranous and endochondral ossification. In stress fractures, which heal through intramembranous ossification, NGF/TrkA signaling triggers reinnervation, vascularization, and osteoblastic activity during repair ${ }^{31}$. Moreover, NGF/TrkA signaling is now known to be crucial in long bone development as well as skeletal adaptations to mechanical loads ${ }^{32,33}$. Together these studies suggest that NGF could be used as an ostebiologic drug to promote long bone fracture repair by synergistically promoting intramembranous and endochondral bone repair. Towards this, our group has detailed the temporal expression of NGF and TrkA during long bone fracture repair and identified the cartilaginous phase as a key timepoint for local exogenous injections of recombinant beta NGF ( $\beta-N G F)$ to accelerate endochondral fracture repair ${ }^{34}$. In this system, $\beta$-NGF delivery promoted cartilage to bone transformation by upregulating the Wnt and Ihh pathways ${ }^{34}$. However, as our published work demonstrated, drug administration by local injection required high dosages and repeated administration to elicit therapeutic effects $^{34}$. Such dosing regimens will likely reduce patient compliance and creates an opportunity to develop controlled delivery platforms that can achieve sustained drug delivery in the absence of surgical implantation ${ }^{35,36}$.

Localized drug delivery to the fracture site with the use of injectable microparticles made of polymeric hydrogels is a highly translatable strategy to overcome the limitations associated with conventional drug administration. Poly (ethylene glycol) (PEG)-based hydrogels are clinically relevant polymer systems based on their low-immunogenic, non-cytotoxic, and biocompatible properties ${ }^{37}$. Therefore, PEG has had extensive biomedical uses for years, particularly drug delivery. Modifying PEG hydrogels with methacrylation (PEGDMA) enables these monomers to be easily formed into three-dimensional (3D) polymers with defined shapes and size through initiation of free radical chain photopolymerization of the methacrylate groups at each end of the PEG chain ${ }^{38}$. Proteins can be readily loaded into PEGDMA microparticles to allow for localized and sustained release, based on the crosslinking density of the polymer ${ }^{39}$. Previously, our group has fabricated high aspect 
ratio PEGDMA microrods by photolithography for targeted drug delivery ${ }^{40,41}$. High-aspect ratio particles, such as microrods, are an exciting class of microparticles that provide a high surface area to volume ratio and form localized 3D porous networks upon injection, which can maximize local cellular interactions and provide open porous systems for localized drug delivery, cellular recruitment, and activation ${ }^{42}$. Additionally, high-aspect ratio microparticles evade internalization by phagocytic cells such as macrophages ${ }^{43,44}$.

In this work, we build on our foundational research showing recombinant $\beta$-NGF promotes endochondral repair in a murine fracture model ${ }^{34}$ by encapsulating the protein in the PEGDMA microrods for sustained local delivery. We hypothesized that local and sustained release of $\beta$-NGF from PEGDMA microrods promotes enhanced endochondral fracture repair relative to soluble $\beta$-NGF and untreated controls. To test this hypothesis, we first aimed to define key characteristics of the PEGDMA microrod polymer mesh network density for maximization of $\beta$-NGF loading efficiencies. By varying concentration of the PEGDMA precursor solution to produce unique network densities, we found that higher \% PEGDMA (v/v) microrods had increased loading efficiencies and more sustained release of $\beta$-NGF over 7 days when compared to lower \% PEGDMA $(\mathrm{v} / \mathrm{v})$ microrods. To confirm $\beta-N G F$ encapsulated within these microrods maintains bioactivity upon release we utilized in vitro studies with erythroleukemia cells, a TrkA expressing cell line, to demonstrate retained cellular proliferation relative to other treatment groups. Subsequently, we established these PEGDMA microrods can be delivered and retained within or adjacent to the fracture callus for at least 7 days post-injection. Efficacy studies using our established murine model demonstrate that the mice receiving $\beta$-NGF loaded PEGDMA microrods exhibited improved fracture repair relative to soluble $\beta$-NGF or negative control. Herein, we describe an injectable system for localized $\beta$-NGF delivery for accelerating endochondral fracture repair.

\section{Methods}

\section{PEGDMA microrod fabrication}

Microrods were fabricated as previously described ${ }^{45}$. Briefly, poly (ethylene glycol) dimethacrylate (PEGDMA molecular weight 750, photoinitiator 2,2-dimethoxy-2- phenylacetophenone (DMPA) were dissolved in 1-vinyln-pyrrolidone (NVP) to a concentration of $100 \mathrm{mg} / \mathrm{mL}$ in phosphate-buffered saline (PBS). 25, 75, and $90 \%$ PEGDMA microrods were created by varying the \% v/v PEGDMA to PBS. Photolithography was used to create microrods designed to have the dimensions $100 \times 15 \times 15 \mu \mathrm{m}$, micro-fabricated on 3-inch silicon wafers. The PEGDMA precursor solution was deposited onto each wafer wherein the wafers had a $15 \mu \mathrm{m}$-deep bevel prefabricated with SU-8 2015. The wafer was exposed using a Karl Suss MJB3 mask aligner to a $405 \mathrm{~nm}$ light source through a microrod patterned photomask at $9 \mathrm{~mW} / \mathrm{cm}^{2}$. Microrods on the wafer were rinsed and removed with $70 \%$ ethanol while gently scraping with a cell scraper. The collected microrods were centrifuged and rinsed in $70 \%$ ethanol three times before being resuspended in sterile deionized water $\left(\mathrm{diH}_{2} \mathrm{O}\right) \mathrm{with}_{10} \%$ sucrose and $0.05 \%$ tween-20 to prevent aggregation. Aliquots of $\sim 100,000$ PEGDMA microrods were then lyophilized, sealed, and stored at $4{ }^{\circ} \mathrm{C}$ until further use. A subset of PEGDMA microrods was resuspended in PBS and micrographed under bright field (BF). Another subset was stained with the low molecular weight dye 4',6-diamidino-2-phenylindole (DAPI) $1 \mu \mathrm{g} / \mathrm{mL}$ in PBS for 5 minutes, washed with PBS gently three times, then immediately imaged using a Nikon Ti microscope.

\section{Protein loading of PEGDMA microrods}

Bovine $\alpha$-Chymotrypsinogen A (Sigma-Aldrich) was used as a proxy for comparing loading efficiencies between 25, 75, and $90 \%$ PEGDMA microrods as its molecular weight $(25.7 \mathrm{kDa})$ is similar to that of $\beta$-nerve growth factor ( $\beta$-NGF, $27 \mathrm{kDa}$ ). Lyophilized PEGDMA microrods aliquots ( 100,000 microrods/aliquot) were resuspended in $20 \mu \mathrm{L}$ of $1 \mathrm{mg} / \mathrm{mL}$-Chymotrypsinogen $\mathrm{A}$ in $\mathrm{diH}_{2} \mathrm{O}$. After resuspension, the microrods were allowed to passively adsorb protein for 30 hours in $4^{\circ} \mathrm{C}$. After loading, microrods were resuspended in $\operatorname{diH}_{2} \mathrm{O}$, gently spun down to pellet in tube, and the supernatants were used to perform a micro bicinchoninic acid $(\mu \mathrm{BCA})$ protein assay to quantify the amount of protein left in solution. To determine loading efficiency, the following equation was used:

Loading efficiency $\%=\left(\frac{X_{i}-X_{t}}{X_{i}}\right) * 100$

Where $X_{i}$ is the quantity of drug added initially during preparation and $X_{t}$ is amount of protein in the supernatant after 30 hours. Loading of PEGDMA microrods with $\beta-N G F$ and calculation of loading efficiency was done similarly in subsequent assays. 


\section{Erythroblast (TF-1) Cell Proliferation}

TF-1 cell proliferation assay was modified from the established method ${ }^{46}$. Briefly, TF-1 cells (ATCC) were cultured for 7 days in RPMI 1640 Medium modified with 2 mM L-glutamine, $10 \mathrm{mM}$ HEPES, 1 mM sodium pyruvate, $4,500 \mathrm{mg} / \mathrm{L}$ glucose, and $1,500 \mathrm{mg} / \mathrm{L}$ sodium bicarbonate (ATCC) supplemented with $2 \mathrm{ng} / \mathrm{ml}$ recombinant human Granulocyte-Macrophage Colony-Stimulating Factor (Sigma-Aldrich) and $10 \%$ fetal bovine serum. Following 7 days of cell growth, confluent TF-1 cells were collected by centrifugation and resuspended into 24-well microplates with 30,000 cells per well containing $600 \mu \mathrm{l}$ of serum-free medium. Cells were cultured in serum-free medium for 24 hours to synchronize the cells prior to adding treatment groups. After 24 hours, high pore density (0.4 micron) transwell inserts containing either $100 \mu \mathrm{l}$ of serum-free medium, 16,000 empty microrods, $2,000 \mathrm{ng}$ of soluble $\beta-\mathrm{NGF}$, or 16,000 microrods containing $18 \mathrm{ng}$ of $\beta$-NGF were inserted into each well and cultured in these conditions for an additional 96 hours. A 24-well plate containing 30,000 cells per well was removed and analyzed (see below) after the 24 hour serum-free medium incubation as a Day 0 control. After 96 hours, the transwell inserts were removed and $300 \mu l$ from each well was aspirated. The $300 \mu \mathrm{l}$ collected was aliquoted into an individual well in a 96-well microplate containing $100 \mu \mathrm{l}$ each ( 3 wells total for a single well in the 24-well plate) and subjected to a CyQuantC Direct Proliferation Assay Kit (Thermo Fisher) per the manufacturers protocol. Data is represented as a fold change relative to the cell number at Day 0.

\section{$\beta$-NGF elution from PEGDMA microrods}

Lyophilized 90 \% PEGDMA microrods were loaded by resuspending 100,000 (100 k) microrod aliquots in 20 $\mu \mathrm{L}$ of $1 \mathrm{mg} / \mathrm{mL}$ recombinant human $\beta-N G F$ (Peprotech) as described in the Protein loading of PEGDMA microrods section. After loading, microrods were gently rinsed thrice with PBS. PEGDMA microrods were then further divided, samples consisted of $16 \mathrm{k}$ microrods/microtube suspended in $250 \mu \mathrm{L}$ of PBS (pH 7.4). Samples placed onto an orbital shaker (100 RPM) within an incubator $\left(37^{\circ} \mathrm{C}\right)$. PEGDMA microrods were spun down gently and the supernatants were collected and replenished at 6, 24, 48, 72, 96, 120, 144, and 168 hours. Collected supernatants were immediately flash frozen and stored at $-80{ }^{\circ} \mathrm{C}$ until further use. ELISAs for human $\beta$-NGF (RayBiotech) were performed per the manufacturer's instructions and daily release amounts were calculated by an established standard curve.

\section{Murine tibial fracture model}

Approval was obtained from the University of California, San Francisco (UCSF) Institutional Animal Care and Use Committee (IACUC) prior to performing the mouse studies and the procedures were carried out in accordance with approved guidelines and regulations. Studies were conducted on the C57BL6/J wild type strain obtained from Jackson Labs. Briefly, adult (10-16 weeks) male mice were anesthetized via inhalant isoflurane, and closed non-stable fractures were made mid-diaphysis of the tibia via three-point bending fracture device ${ }^{47}$. Fractures were not stabilized as this method promotes robust endochondral repair. After fractures are created, animals were provided with post-operative analgesics (buprenorphine sustained release). Animals were socially housed and allowed to ambulate freely.

\section{Local injections}

Percutaneous injections into tibial fracture calluses of mice were administered 7 days post-fracture. A precise microliter Hamilton@ syringe was utilized for all injections wherein experimental agents were injected in $20 \mu \mathrm{L}$ of PBS. Experimental groups are as follows: Controls injected with sterile PBS, $\beta$-NGF group injected with 500 ng of $\beta$-NGF in PBS, non-loaded microrods group injected with 16,000 PEGDMA microrods in PBS, and $\beta-$ NGF microrods group injected with 16,000 PEGDMA microrods loaded with $18 \mathrm{ng}$ of $\beta$-NGF.

\section{Micro-Computed tomography $(\mu \mathrm{CT})$}

$\mu C T$ analysis was performed as previously described ${ }^{48,49}$. Fracture tibias were dissected free of attached muscle 14 days post-fracture, fixed in $4 \%$ PFA and stored in $70 \%$ ethanol. Fracture calluses were analyzed in the UCSF Core Center for Musculoskeletal Biology (CCMBM, NIH P30 funded core) using the Scanco $\mu$ CT50 scanner (Scanco Medical AG, Basserdorf, Switzerland) with $10 \mu \mathrm{m}$ voxel size and X-ray energies of $55 \mathrm{kVp}$ and $109 \mu \mathrm{A}$. A lower excluding threshold of $400 \mathrm{mg}$ hydroxyapatite $(\mathrm{HA}) / \mathrm{mm}^{3}$ was applied to segment total mineralized bone matrix from soft tissue in studies of control and treated mice. Linear attenuation was calibrated using a Scanco hydroxyapatite phantom. The regions of interest (ROI) included the entire callus without existing cortical clearly distinguished by its anatomical location and much higher mineral density. $\mu C T$ reconstruction and quantitative analyses were performed to obtain the following structural parameters: volume 
fraction (bone volume/total volume as \%), trabecular connective density as trabecular bifurcations $\left(\# / \mathrm{mm}^{3}\right)$, and bone mineral density $\left(\mathrm{mg} \mathrm{HA} / \mathrm{cm}^{3}\right)$.

\section{Localization histology}

Tibias were harvested 12,14 , and 21 days post-fracture $(5,7$, or 14 days post-injection of microrods) to observe microrod localization. At time of collection, tibias were fixed in $4 \%$ PFA and decalcified in $19 \%$ EDTA for 14 days at $4{ }^{\circ} \mathrm{C}$ with rocking and solution changes every other day. Tibia were processed for paraffin embedding, serial sections were cut at $10 \mu \mathrm{m}$ (3 sections per slide), and Hall-Brundt's Quadruple (HBQ) staining protocol was done to visualize the bone (red) and cartilage (blue) as previously described to localize PEGDMA microrods ${ }^{34,49}$. The sections were mounted on slides with Permount ${ }^{\mathrm{TM}}$ mounting medium and brightfield images were captured on a Leica DMRB microscope.

\section{Histomorphometry}

Fracture callus composition was determined using quantitative histomorphometry of tibia harvested 14 days post-fracture. Standard principles of histomorphometric analysis ${ }^{49}$ were utilized to quantify the bone and cartilage fraction in the fracture callus using the first section from every $10^{\text {th }}$ slide analyzed, such that sections were $300 \mu \mathrm{m}$ apart. Images were captured using a Nikon Eclipse Ni-U microscope with Nikon NIS Basic Research Elements Software version 4.30. Quantification of callus composition (cartilage, bone, fibrous tissue, background) was determined using the Trainable Weka Segmentation add-on in Fiji ImageJ (version 1.51.23; $\mathrm{NIH}$, Maryland, USA $)^{50}$. Volume of specific tissue types was determined in reference to the entire fracture callus by summing the individual compositions relative to the whole.

\section{Statistical analysis}

Individual dots on graphs represent biological replicates, error bars represent standard error of the mean (SEM). Measurements were taken from distinct samples. Data were analyzed using GraphPad Prism (version 8, GraphPad Software, San Diego, CA). ANOVA was used to determine statistical differences between multiple groups followed by Tukey's HSD post-hoc comparison testing. Significant differences were defined at $p<0.05$.

\section{Results}

\section{Injectable PEGDMA microrod fabrication via photolithography}

PEGDMA microrods were fabricated through a process of photolithography (Fig. 1). The exact dimensions of the PEGDMA microrods can be carefully controlled by the photomask (length and width). The microrod height is determined by distance between the silicon wafer and the photomask which is manually controlled by use of the mask aligner. PEGDMA microrods are then cross-linked with UV irradiation, detached with a cell scraper from the silicon wafer and collected. Each individual PEGDMA microrod had the following dimensions: $H=15$ $\mu \mathrm{m}, W=15 \mu \mathrm{m}$, and $L=100 \mu \mathrm{m}$. The 3D structure of the microrods is formed through free radical chain photopolymerization of the methacrylate groups at each end of each 750 MW PEG monomer unit. This system provides a high-throughput method to produce highly uniform PEGDMA microrods.

\section{PEGDMA microrod macromer concentration changes protein-loading efficiency}

The first goal was to tune the PEGDMA microrod polymer network density to maximize protein loading efficiency. Prior to photolithography, the PEGDMA macromer concentration was adjusted to contain low (25 $\%$ ), medium (75\%), or high (90\%) volume (v/v) amounts (Fig. 2A). After fabrication, the PEGDMA microrods were lyophilized (freeze dried) to remove any residual liquid remaining that could alter protein loading and then loaded with $\alpha$-Chymotrypsinogen $A$ as a proxy protein, as its molecular weight is similar to that of $\beta$-NGF (26 $\mathrm{kDa}$ ). The low and medium macromer volume PEGDMA microrods had modest loading efficiencies of less than $5 \%$ and $15 \%$, respectively. The high macromer volume PEGDMA microrods exhibited a significantly larger loading efficiency, over $30 \%$, when compared to the other PEGDMA concentrations. Since the $90 \%$ PEGDMA (v/v) microrods had the best loading efficiency, we chose this formulation for all the subsequent experiments. We then confirmed $\beta$-NGF loading efficiency by loading high macromer PEGDMA microrods with $\beta$-NGF which resulted in over $40 \%$ loading efficiency (Supplemental Fig. 1). DAPI, a commonly used nuclear counter stain, can be easily adsorbed into the PEGDMA microrods and visualized with fluorescent microscopy (Fig. 2B). The DAPI-stained microrods are uniform in size and do not exhibit any aggregation, indicating good dispersity in solutions to allow for more optimal protein loading. Given that protein loading is driven by 
physisorption, we wanted to qualitatively examine the absorption of proteins onto the microrods and the rate at which protein elution occurs after loading. Using FITC-BSA as a model protein, the superficial layer of the PEGDMA microrods are coated with the fluorescently tagged protein with no diffusion at time 0 (Fig. 2C, Top). After 60 minutes of incubation, diffusion is drastically increased and FITC-BSA can be observed eluting in the surrounding space of the PEGDMA microrods (Fig. 2C, Bottom).

\section{Bioactivity retention and sustained release of $\beta$-NGF from PEGMDA microrods}

We next tested whether $\beta$-NGF retained bioactivity when released from the PEGDMA microrods. To do this, we utilized the erythroleukemia TrkA expressing cell line, TF-1, in the presence of culture media (control), $2,000 \mathrm{ng}$ of soluble $\beta$-NGF, non-loaded microrods, or 16,000 PEGDMA microrods loaded with $18 \mathrm{ng}$ of $\beta$-NGF (Fig. 3A). We hypothesized that sustained release from $\beta-N G F$ microrods would increase proliferation of TF-1 cells relative to the other treatment groups following 4 days of culture. As expected, soluble $\beta$-NGF treated cells exhibited a 2-fold increase in proliferation relative to the control. Interestingly, the non-loaded microrods also exhibited a similar increase in proliferation as soluble $\beta-N G F$ treated cells. However, our hypothesis was supported by the statistically significant 4 -fold increase in proliferation by the $\beta$-NGF microrods. The increase in proliferation may be attributed to the sustained release of $\beta$-NGF which we observed over a 168 hour (7 day) period (Fig. 3B \& C). The $\beta-N G F$ microrods exhibited an initial burst release of $\beta-N G F$ within the first 24-48 hours, followed by sustained release over the next 120 hours (days 2-7), as measured by ELISAs (Fig. 3B). The total daily amount of eluted $\beta$-NGF decreased with time indicating concentration dependent (first order) release kinetics from the PEGDMA microrods. Nonetheless, we were able to detect and quantify elution of $\beta$ NGF over a 7 day period (168 hours) (Fig. 3C).

\section{B-NGF loaded PEGDMA microrods promote endochondral bone formation}

Our data thus far has demonstrated that PEGDMA microrods can be loaded with $\beta$-NGF and $\beta$-NGF is bioactive and released over a 7 day period. Given these findings, we hypothesized that sustained release of $\beta$ NGF loaded PEGDMA microrods could accelerate endochondral fracture repair. To test this hypothesis, we utilized a murine model of long bone healing wherein closed, mid-shaft fractures were created in the right tibia of adult wild type mice (Supplemental Fig. 2). As previously shown, these non-stabilized fractures incite robust endochondral repair ${ }^{34,49,51}$. In our previous study, we demonstrated that $\beta$-NGF was most effective in promoting fracture repair when delivered 7 days post-injury, during the cartilaginous phase of bone healing ${ }^{34}$. Based on this we chose to similarly deliver PEGDMA microrods 7 days post injury using a Hamilton syringe for percutaneous directly to the fracture site. First, we confirmed that the 16,000 microrods suspended in $20 \mu \mathrm{L}$ saline (lightly stained blue) could effectively be delivered to fracture callus (Fig. 4A-D) and remained localized throughout the entirety of the repair period of 14 days (Fig. 4C-E) as visualized by Hall-Brunt's Quadruple $(\mathrm{HBQ})$ staining $($ cartilage $=$ blue, bone $=$ red). To assess the effectiveness of $\beta$-NGF, 16,000 microrods containing $18 \mathrm{ng}$ of $\beta$-NGF was injected into the fracture callus. For comparison, additional mice were divided into three experimental (injection) groups: fracture calluses were percutaneously injected with either $20 \mu \mathrm{L}$ saline, single dose of $\beta$-NGF (2,000 ng), or non-loaded PEGDMA microrods. All percutaneous injections were administered 7 days post-fracture and were allowed to heal for 7 days (14 days post-fracture) at which point the calluses were harvested for Micro-Computed tomography $(\mu \mathrm{CT})$ analysis to quantify the mineralized tissue within the fracture callus and to analyze the bone tissue microarchitecture. By gross examination of the images, the $\beta$-NGF microrods group appeared to have a largest most consolidated bony callus compared to all others (Fig. 5A-D). Quantification of the bone volume fraction (BFV) confirmed the highest BVF in the $\beta$-NGF microrods group with a significantly higher BVF ( $\sim 2 \%$ increase) compared to saline controls (Fig. 5E). Additionally, the fractures treated with $\beta$-NGF microrods resulted in more mature fracture calluses. $\beta-N G F$ microrods treatment significantly increased trabecular bifurcations (TB, 95\% increase) and bone mineral density (BMD, $\sim 34 \%$ increase) compared to saline controls (Fig. 5F-G). Interestingly, the soluble $\beta-N G F$ did not improve bone formation to the same extent as $\beta$-NGF microrods and was not statistically different from the saline controls. Although not statistically different, the non-loaded microrods exhibit higher amounts of BVF, TB, and BMD when compared to the soluble $\beta-N G F$ and saline treated groups.

\section{B-NGF loaded PEGDMA microrods reduce cartilaginous tissue volume in the fracture callus.}

To understand the differences noted by $\mu \mathrm{CT}$ at a more detailed tissue level, we utilized quantitative histology to differentiate the cartilage and bone fractions within the fracture callus. Histological images of tibia sections harvested 14 days post-fracture stained with $\mathrm{HBQ}$ (cartilage $=$ blue, bone $=$ red) visually indicate that $\beta-N G F$ microrods have the highest amount of bone (Fig. 6A-D). Saline treated fracture calluses had the high 
quantities of cartilage as percent composition of the callus (32 $\pm 2 \%)$ with the least bone volume as percent composition (67 $\pm 2 \%$ ) compared to other treatment groups indicating the least advanced healing (Fig. 6A-F). Higher magnification images verify large proportions of chondrocytes in the fracture callus which suggest the fracture is only nearing the cartilage to bone transition phase in endochondral repair (Fig. 6A). Near identical results were observed for the empty microrod treated fracture calluses with cartilage and bone volume at $32 \pm$ $2.7 \%$ and $68 \pm 2.7 \%$, respectively (Fig. $6 \mathrm{C}$ and $6 \mathrm{E}-\mathrm{F}$ ). The soluble $\beta-N G F$ treated fracture calluses resulted in slightly elevated levels of bone $(71 \pm 3.2 \%)$ and lowered cartilage volumes $(29 \pm 3.2 \%)$ relative to the empty microrods and untreated controls, but this effect was not statistically different. $\beta$-NGF loaded microrods were the only treatment group to significantly change the fracture callus composition producing robust bone formation (79 $\pm 3 \%$ ) (Fig. 6D and 6F). $\beta-N G F$ microrod treated samples also show a significant visual reduction in cartilage and statistically different cartilage volume $(21 \pm 3 \%)$ compared to saline controls (Fig. 6D and 6E).

\section{Discussion}

Recent work in the field supports that NGF signaling plays a significant role in both intramembranous and endochondral ossification. The pioneering mechanistic studies first describe a critical role of NGF/TrkA signaling during skeletal development and adaptation to mechanical load ${ }^{32,33}$. Subsequently our group delineated the spatiotemporal expression of TrkA during long bone fracture repair and identified the cartilaginous phase of healing as a key timepoint for exogenous $\beta$-NGF injections by promoting pathways associated with endochondral ossification ${ }^{34}$. Most recently, NGF/TrkA signaling was also shown to be acutely upregulated following stress fracture, triggering reinnervation, vascularization, and osteoblastic activity ${ }^{31}$. Stress fractures are a specific and unique subclass of fracture repair that heal through intramembranous bone formation. Together these studies suggest that NGF could be used as an osteobiologic drug to promote long bone fracture repair by synergistically promoting intramembranous and endochondral bone repair.

This study aimed to build on our previous work by developing a clinically relevant drug delivery system for sustained $\beta$-NGF delivery using PEG-based hydrogel microparticles. In our previous study, we locally injected $500 \mathrm{ng}$ of recombinant $\beta$-NGF once daily for 3 days to produce a quantifiable acceleration of fracture healing. However, $\beta$-NGF is a known hyperalgesic and preclinical studies utilizing murine models have demonstrated that hyperalgesia in mice can be experienced with daily injections of $100 \mathrm{ng}$ and above $\mathrm{e}^{52,53}$. Thus, our goal is to balance the trophic benefit of $\beta-N G F$ therapy while minimizing its hyperalgesic effects by providing sustained drug release at a dosing below this threshold. To avoid repeated doses, we aimed to use PEGDMA microrods as a clinically relevant drug delivery platform. The majority of PEGDMA microparticle delivery platforms use spherical particles for bone repair applications ${ }^{54-56}$. Uniquely, in our study we demonstrate the use of high aspect ratio microrods for fracture repair given that high aspect ratio particles have higher residence time and tend to evade phagocytosis or cellular internalization ${ }^{43,44}$. To our knowledge, this is the first study to use PEGDMA microrods for bone fracture repair.

By employing photolithography, PEGDMA microrods can be produced in a high throughput fashion. Moreover, we were able to increase $\beta$-NGF loading onto PEGDMA microrods using $90 \%$ (v/v) PEGDMA macromer. Tuning the cross-linking densities can alter the polymer mesh size within the hydrogel and subsequent drug loading and release ${ }^{36,39}$. Higher concentrations of PEG have shown greater loading capacities as seen in our system ${ }^{55}$. Interestingly, striking visual differences in loading are apparent between lower and higher molecular weight molecules such as DAPI and FITC-BSA. DAPI stains the PEGDMA microrods uniformly versus the FITC-BSA that can mostly be visualized on the surface of the PEGDMA microrods immediately after loading. Given that DAPI has a molecular weight of $0.277 \mathrm{kDa}$ compared to the $67 \mathrm{kDa}$ size of FITC-BSA, the micrographs confirmed that molecules of smaller size can more readily diffuse across or into the PEGDMA polymer mesh network.

We next wanted to demonstrate that the loaded $\beta$-NGF retained its bioactivity with an in vitro proliferation assay using the TrkA expressing TF-1 cell line ${ }^{46}$. Importantly, this assay was performed with 16,000 PEGDMA microrods, versus 100,000 used for the loading assay. This is because only 16,000 could effectively be aspirated in a $20 \mu \mathrm{L}$ syringe used for the in vivo experiments. We calculated that approximately $30-40 \%$ of total protein loaded in 100,000 microrods was about 1-2 mg. Therefore, we used the highest calculation of $2 \mu \mathrm{g}$ $(2,000 \mathrm{ng})$ to be loaded into the 16,000 microrods and set that as our soluble $\beta$-NGF amount for all 
experiments in parallel. However, the highly specific ELISA assay measured only $18 \mathrm{ng}$ of $\beta$-NGF in 16,000 PEGDMA microrods. This finding suggests that some of the $\beta$-NGF proteins may lose their native molecular arrangement during loading or elution, thus reducing bioactivity. Presumably, the discrepancy between the $\mu \mathrm{BCA}$ assay and the ELISA calculations may be attributed to the disruption of $\beta$-NGF's non-covalent homodimer confirmation or the non-specificity of the $\mu \mathrm{BCA}$ assay. Nonetheless, 16,000 PEGDMA microrods containing $18 \mathrm{ng}$ of bioactive $\beta$-NGF's had a potent effect on TF-1 cell proliferation. This potent effect is likely driven by the sustained release of $\beta$-NGF over the 96 hour experimental period. We also observed a nominal increase in proliferation of cells cultured with non-loaded PEGDMA microrods. Although the degradation products of the PEGDMA microrods were not evaluated in this study, PEG at low concentrations have previously been shown to slightly elevate cell proliferation which could be contributing to TF-1 cell proliferation in this study ${ }^{57}$.

Next, we examined PEGDMA microrod localization during endochondral fracture repair in a murine fracture model and were able to histologically localize the PEGDMA microrods at both 5 and 7 days post-injection. However, the PEGDMA microrods were no longer visible after 14 days suggesting that the PEGDMA microrods are perhaps physically degraded overtime due to the dynamic mechanical microenvironment within the fracture callus ${ }^{58}$. Additionally, the fractured leg experiences regular loading due to free ambulation of the animals, which could also contribute to mechanical degradation of the PEGDMA microrods. Degradation products of the PEGDMA microrods are not of concern in vivo based on established biocompatibility and noncytotoxicity ${ }^{38,40,41}$. Importantly, efficacy studies confirm that the PEGDMA microrods were localized in the fracture callus long enough to deliver the therapeutic payload and therefore are suitable for application in fracture callus injections of $\beta-N G F$.

Therapeutic efficacy of $\beta$-NGF delivery via PEGDMA microrods was validated using non-stabilized tibial fracture in mice followed by $\mu \mathrm{CT}$ analysis and quantitative histomorphometry. $\beta$-NGF loaded microrods enhanced endochondral fracture repair as evidenced by the reduction in cartilage volume and statistically significant increases in bone volume fraction (BVF), trabecular bifurcations (TB), and bone mineral density (BMD). Notably, the woven-like bone morphology and minimal hypertrophic chondrocyte cells within the fracture callus further indicates a quicker transition into the bony callus formation after injection with sustained release $\beta$-NGF loaded PEGDMA microrods, supporting our hypothesis. We ascertain that this is likely due to the sustained release of $\beta-N G F$ from the PEGDMA microrods versus a large bolus dose from free/injected $\beta$ NGF. Large bolus doses are at risk of off target effects and toxicity ${ }^{12,35,39}$. Thus, sustained release from hydrogels provides a more optimal strategy primarily from a pharmacokinetic approach by slowly delivering drug and maintaining a high local concentration of drug in the target tissue ${ }^{36,59}$.

Another key aspect that could be contributing to the enhanced endochondral bone repair is the likelihood of an increased half-life of $\beta$-NGF within the PEGDMA microrods. Hydrogel delivery systems have been described in the literature to improve the half-life of drugs and biologics ${ }^{39,60,61}$. Specifically, $\beta-N G F$ has an extremely fast distribution half-life of 5.4 minutes and an elimination half-life of 2.3 hours. ${ }^{62}$ Thus, we speculate that the halflife of the successfully loaded $\beta-N G F$ is extended by encapsulation into the PEGDMA microrods. One important distinction to note is the large difference in $\beta$-NGF used in the experimental groups; the soluble $\beta$ NGF dose $(2,000 \mathrm{ng})$ was $\sim 111$-fold larger than the $\beta$-NGF microrods (18 $\mathrm{ng})$. However, despite the markedly lower dose of $\beta-N G F$, the loaded PEGDMA microrods significantly promoted endochondral bone repair compared to the saline treated mice. In addition to potentially extending the half-life of $\beta$-NGF, the high localization of $\beta$-NGF provided by the PEGDMA microrods may synergistically have contributed to the robust endochondral fracture response seen in mice treated with $\beta$-NGF loaded microrods.

\section{Conclusions}

We have demonstrated the feasibility of using PEGDMA microrods for $\beta$-NGF sustained delivery and its therapeutic efficacy in a preclinical murine fracture model. We build on our previous data that showed repeated injections of $\beta$-NGF during fracture repair promotes endochondral fracture repair. Although that study used multiple injections, this study suggests that lower doses (18 $\mathrm{ng}$ ) may in fact be more beneficial than larger doses when $\beta-N G F$ is delivered locally in a sustained manner and encapsulated in a delivery system that may increase the biologics half-life. This dosing regimen of $18 \mathrm{ng}$ is clinically relevant given that NGF has known 
hyperalgesic effects and thus limiting the total amount of NGF could potentially reduce the pain experienced by subjects. From a therapeutic standpoint, reducing the pain and number of injections is critical given that increased stress can lead to delayed fracture healing ${ }^{67}$. To deliver a sustained dose of $\beta$-NGF we utilized PEGDMA hydrogels, as PEG and PEG-conjugates have been FDA approved in the past for several products from tissue adhesives to particle based drug and gene delivery systems ${ }^{68-70}$. To that end, the use of injectable $\beta$-NGF loaded PEGDMA microrods validates a novel and translational therapeutic approach for improving bone fracture repair.

\section{Acknowledgements}

The study was supported by National Institutes of Health through NIGMS (R25-GM056847 to K.O.R.), NIDCR (F31-DE028485 to K.O.R., F30-DE031158 to D.L.C.), NIAMS (R01-AR077761 to C.S.B, T.M., and T.A.D.) and the UCSF Academic Senate Committee on Research to C.S.B. and T.A.D. The content is solely the responsibility of the authors and does not necessarily represent the official views of the National Institutes of Health. We would like to thank Nick Szeto and Dr. Wenhan Chang at UCSF's Core Center for Musculoskeletal Biology \& Medicine for $\mu$ CT analysis.

\section{Author Contributions}

K.O.R, C.S.B., and T.A.D. designed the experiments. K.O.R, B.N.K., and A.N.K. were involved with microfabrication. K.O.R., D.L.C., and A.N.K., performed experiments. K.O.R., D.L.C, V.D., and K.M.O. analyzed the data. K.O.R., D.L.C, C.S.B., and T.A.D. interpreted the results and wrote the manuscript. K.O.R. and D.L.C contributed equally to the manuscript. K.O.R., D.L.C., T.M, C.S.B., and T.A.D. received funding. 


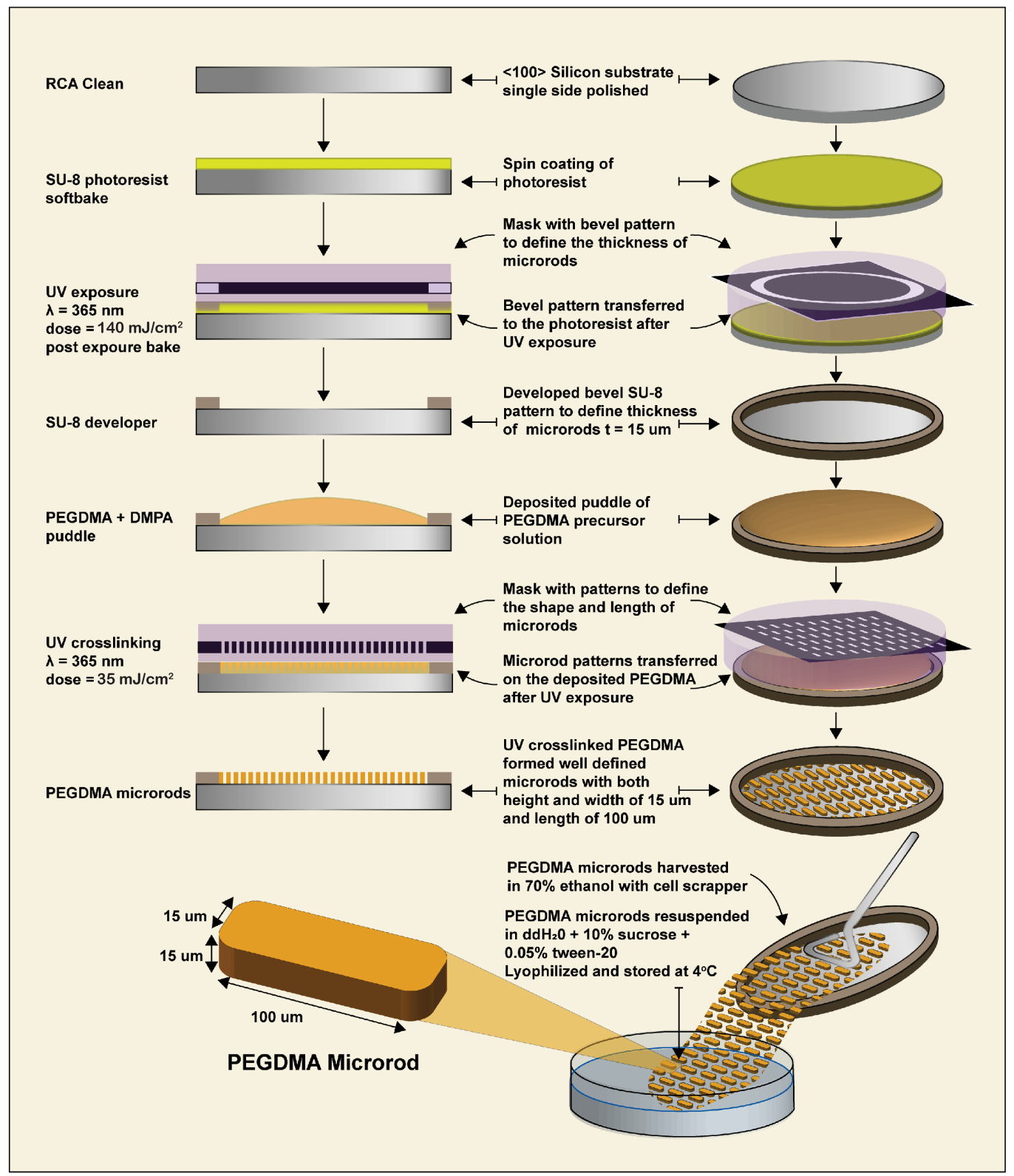

Figure 1. Schematic of PEGDMA microrod fabrication via photolithography. 

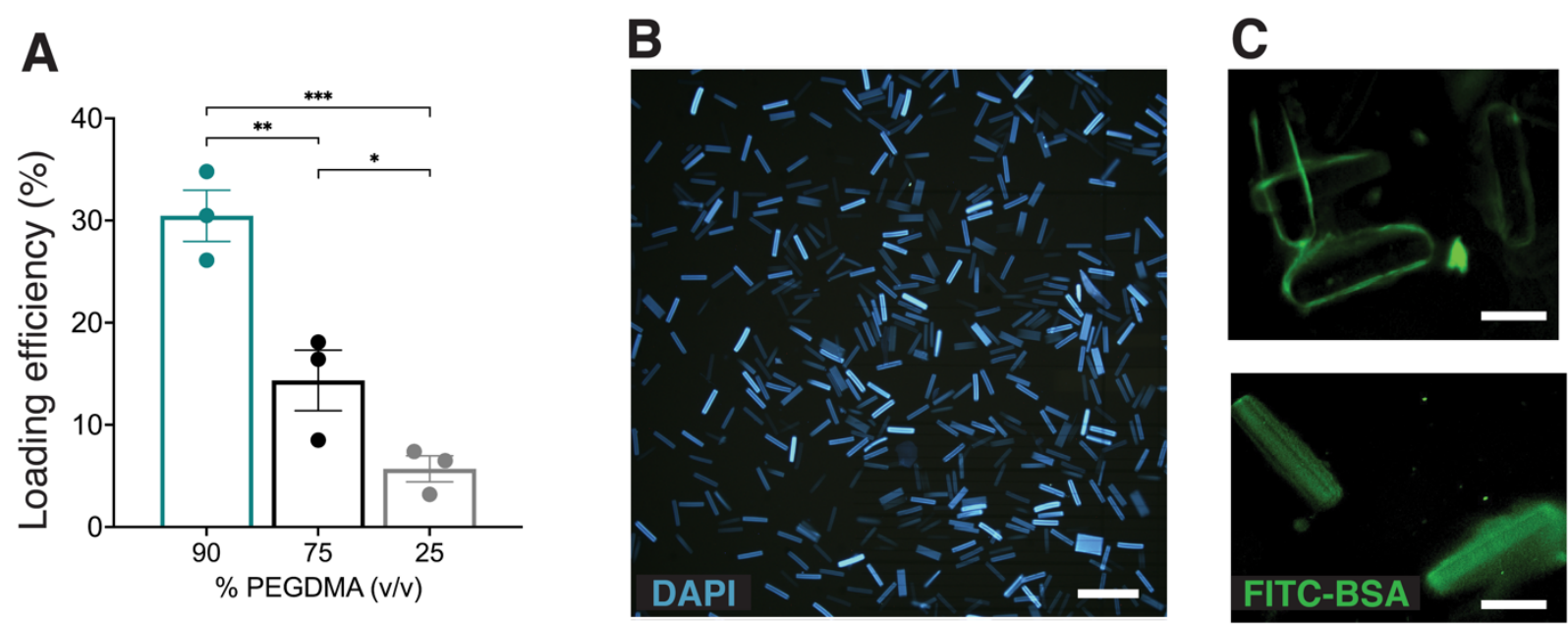

Figure 2. Lyophilized PEGDMA microrods are readily protein loaded. (A) Loading efficiency significantly increases with increasing concentration of PEGDMA (\% PEGDMA v/v). Data shown as means with error bars representing SEM. ${ }^{*} p<0.05,{ }^{* *} p<0.01, * * * p<0.001$ determined by ANOVA with Tukey's post hoc test for multiple comparisons $(n=3)$ (B) DAPI-loaded 90\% PEGDMA microrods, scale bar $=25 \mu \mathrm{m}$. (C) Fluorescent micrographs of FITC-BSA loaded in 90\% PEGDMA microrods taken after 0 mins (top) and after 60 mins of incubation at room temperature (bottom), scale bars $=50 \mu \mathrm{m}$. 

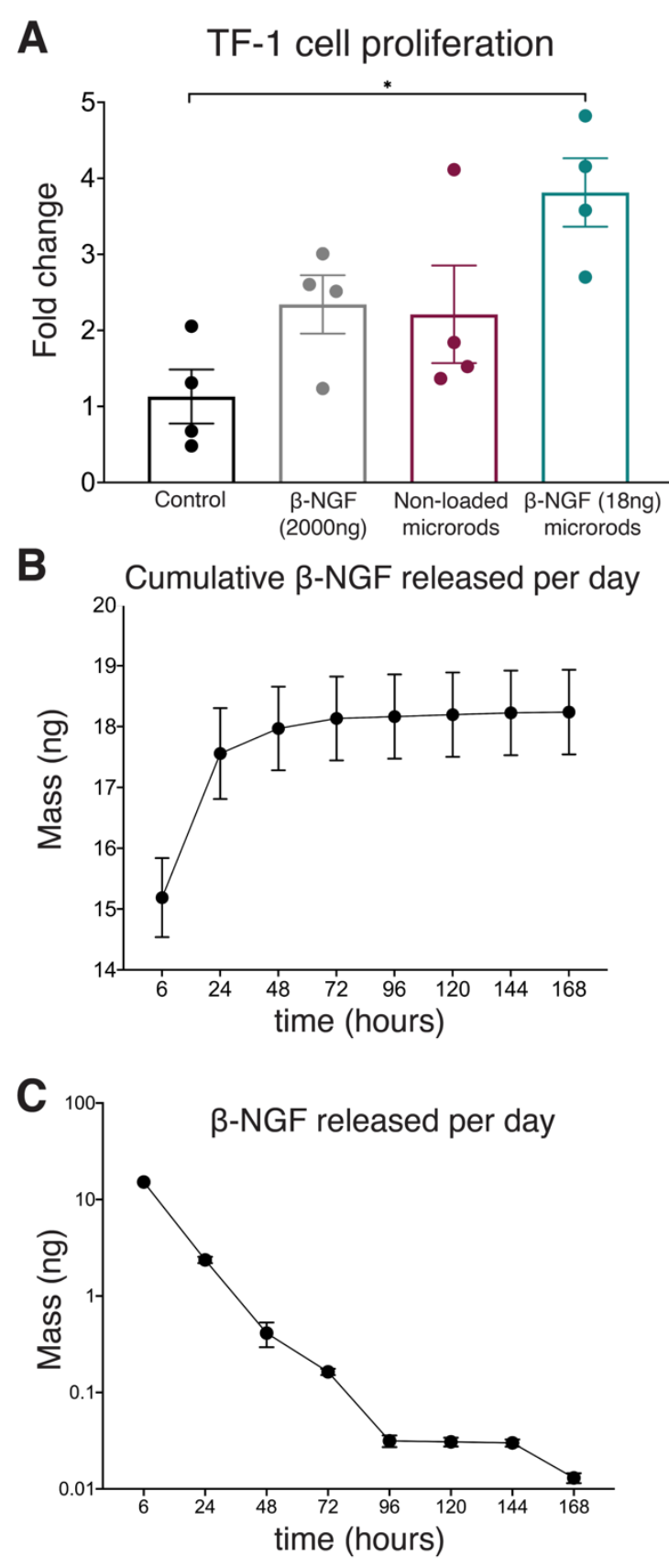

Figure 3. $\beta$-NGF loaded onto PEGDMA microrods retain bioactivity. (A) Relative fold change in TF-1 cell proliferation (day 4 vs day 0 ) for each experimental group. ${ }^{*} p<0.05$ determined by ANOVA with Tukey's post hoc test for multiple comparisons $(n=4)$. (B) Cumulative mass (in $n g$ ) and (C) daily mass (in $n g$ ) of beta NGF released from 90\% PEGDMA microrods over a 7-day period shown in hours $(n=4)$. All data shown as means with error bars representing SEM. 

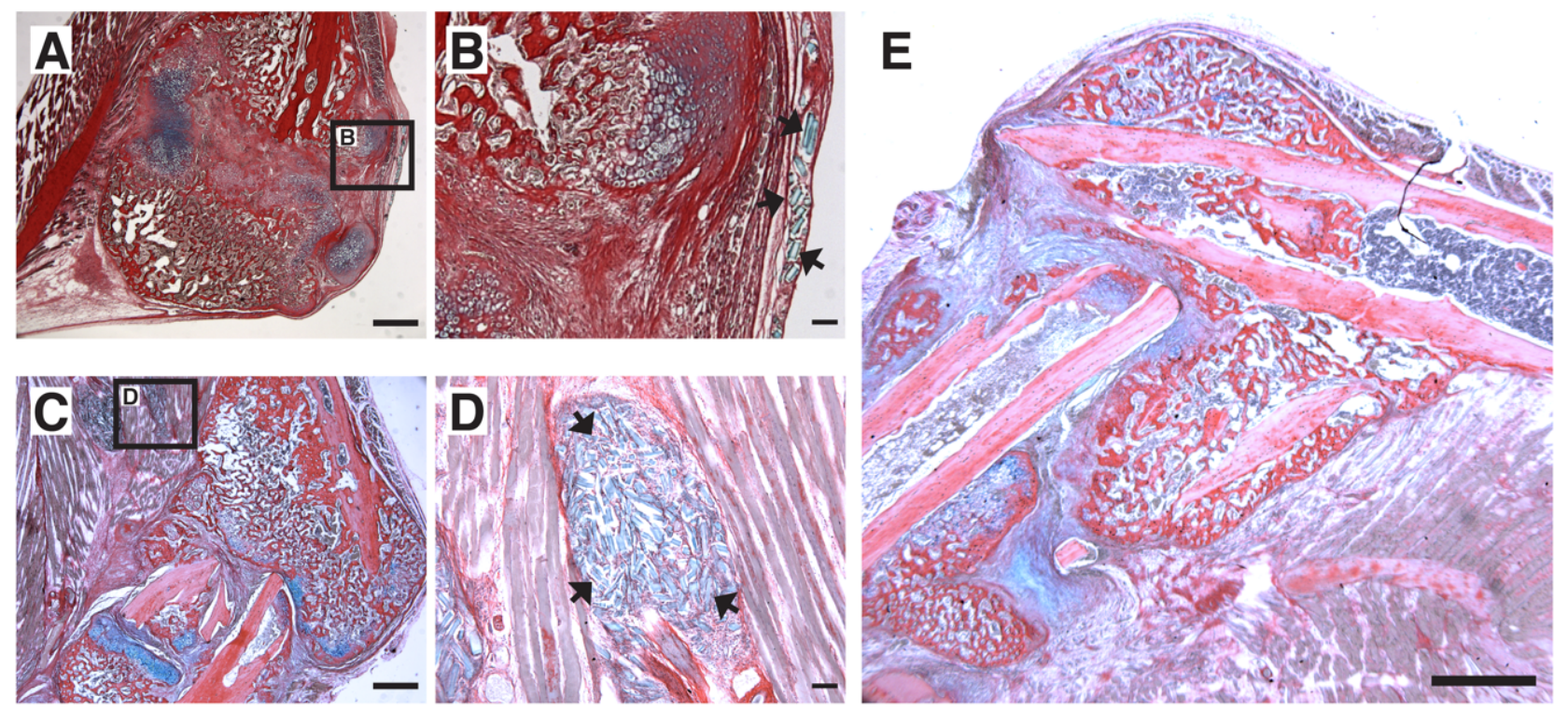

Figure 4. Localization of PEGDMA microrods within tibial fracture calluses. Representative micrographs of (A) low and (B) high magnification of HBQ-stained fracture calluses 5 days postmicrorod injection. Representative micrographs of (C) low and (D) high magnification of fracture calluses 7 days post-microrod injection. Arrows indicate PEGDMA microrods within calluses. $(A, C)$ Scale bars $=1 \mathrm{~mm},(B, D)$ Scale bars $=100 \mu \mathrm{m}$. $(E)$ Representative micrograph of fracture callus 14 days post-injection, scale bar $=1 \mathrm{~mm}$. 


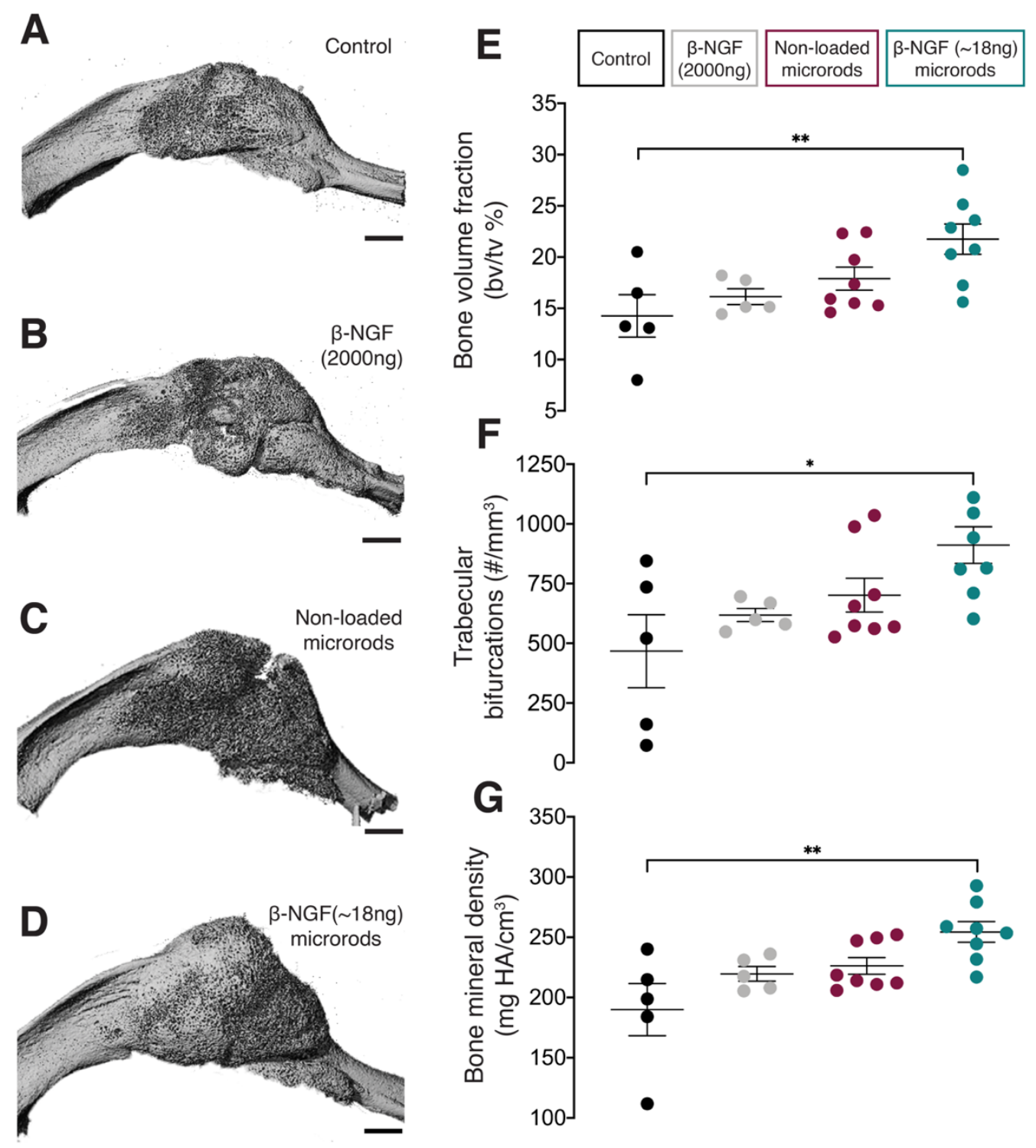

Figure 5. MicroCT analysis of newly formed bone within fracture calluses. Representative three-dimensional images of tibial fracture calluses from mice treated 14 days post-fracture with (A) saline as controls (B) single dose of $\beta-N G F$ (2000ng) (C) non-loaded PEGDMA microrods and (D) PEGDMA microrods loaded with $\beta$-NGF (18ng). Scale bars $=1 \mathrm{~mm}$. Quantification of $(E)$ bone volume fraction $(F)$ trabecular connective density and $(G)$ bone mineral density. Error bars represent $S E M, *_{p}<0.05$, $* *_{p}<0.01$ determined by ANOVA with Tukey's post hoc test for multiple comparisons. 


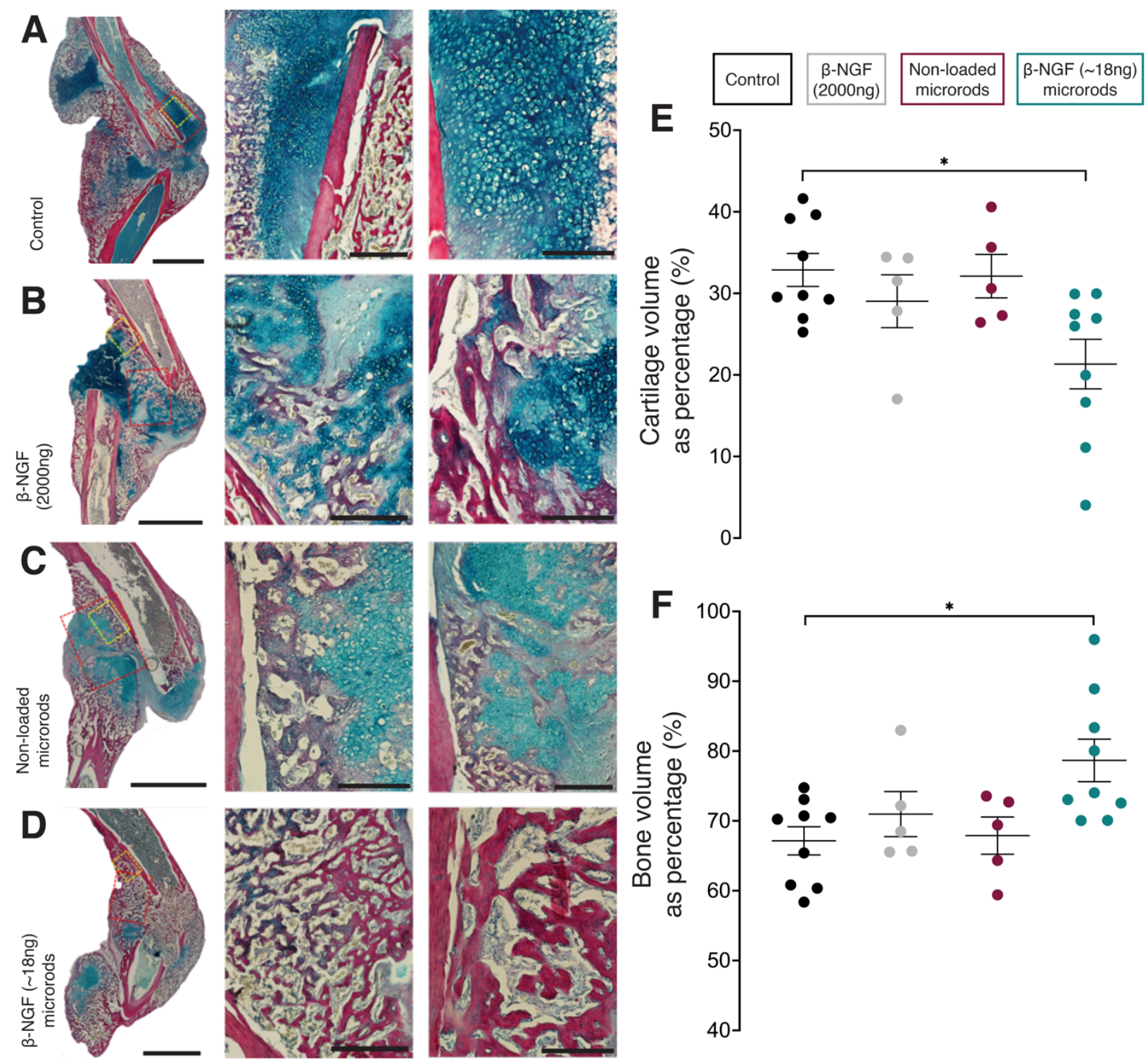

Figure 6. Single injections of PEGDMA microrods loaded with $\beta$-NGF promote endochondral bone repair. Representative micrographs of $\mathrm{HBQ}$-stained fracture calluses from mice 14 days post-fracture treated with (A) saline as controls (B) single dose of $\beta-N G F(2000 n g)$ (C) nonloaded PEGDMA microrods and (D) PEGDMA microrods loaded with $\beta$-NGF (18ng). Left column scale bars $=2 \mathrm{~mm}$, middle and right column scale bars $=500 \mu \mathrm{m}$. Quantification of (E) cartilage volume and (F) bone volume, both given as percent composition of fracture callus. Error bars represent SEM, * $p<0.05$ determined by ANOVA with Tukey's post hoc test for multiple comparisons. 
Literature Cited:

1. United States Bone and Joint Initiative: The Burden of Musculoskeletal Diseases in the United States (BMUS), Third Edition. (2014).

2. Baim, S. \& Leslie, W. D. Assessment of Fracture Risk. Current Osteoporosis Reports 10, $28-41$ (2012).

3. Bonafede, M., Espindle, D. \& Bower, A. G. The direct and indirect costs of long bone fractures in a working age US population. Journal of Medical Economics 16, 169-178 (2013).

4. Marsell, R. \& Einhorn, T. A. Emerging Bone Healing Therapies. Journal of Orthopaedic Trauma 24, (2010).

5. Penn-Barwell, J. G. et al. Severe open tibial fractures in combat trauma. The Bone \& Joint Journal 95-B, 101-105 (2013).

6. Dickson, K. F., Katzman, S. \& Paiement, G. The importance of the blood supply in the healing of tibial fractures. Contemporary orthopaedics 30, 489-493 (1995).

7. Khan, S. N. et al. The Biology of Bone Grafting. JAAOS - Journal of the American Academy of Orthopaedic Surgeons 13, (2005).

8. Roberts, T. T. \& Rosenbaum, A. J. Bone grafts, bone substitutes and orthobiologics. Organogenesis 8, 114-124 (2012).

9. Lukač, N. et al. What do we know about bone morphogenetic proteins and osteochondroprogenitors in inflammatory conditions? Bone 137, 115403 (2020).

10. Food \& Administration, D. Summary of Safety and Effectiveness Data. (2004).

11. James, A. W. et al. A Review of the Clinical Side Effects of Bone Morphogenetic Protein-2. Tissue engineering. Part B, Reviews 22, 284-297 (2016).

12. Food \& Administration, D. FDA Public Health Notification: Lifethreatening Complications Associated with Recombinant Human Bone Morphogenetic Protein in Cervical Spine Fusion. (2008).

13. Bahney, C. S. et al. Cellular biology of fracture healing. Journal of Orthopaedic Research 37, 35-50 (2019).

14. Wong, S. A. et al. Microenvironmental Regulation of Chondrocyte Plasticity in Endochondral Repair-A New Frontier for Developmental Engineering . Frontiers in Bioengineering and Biotechnology vol. 658 (2018). 
15. Bahney, C. S., Hu, D. P., Miclau, T. \& Marcucio, R. S. The Multifaceted Role of the Vasculature in Endochondral Fracture Repair . Frontiers in Endocrinology vol. 64 (2015).

16. Kostenuik, P. \& Mirza, F. M. Fracture healing physiology and the quest for therapies for delayed healing and nonunion. Journal of Orthopaedic Research 35, 213-223 (2017).

17. Kronenberg, H. M. Developmental regulation of the growth plate. Nature 423, 332-336 (2003).

18. Tsang, K. Y., Tsang, S. W., Chan, D. \& Cheah, K. S. E. The chondrocytic journey in endochondral bone growth and skeletal dysplasia. Birth Defects Research Part C: Embryo Today: Reviews 102, 52-73 (2014).

19. Wang, W., Rigueur, D. \& Lyons, K. M. TGF $\beta$ signaling in cartilage development and maintenance. Birth Defects Research Part C: Embryo Today: Reviews 102, 37-51 (2014).

20. Cheng, A. \& Genever, P. G. SOX9 determines RUNX2 transactivity by directing intracellular degradation. Journal of Bone and Mineral Research 25, 2680-2689 (2010).

21. Mak, K. K., Kronenberg, H. M., Chuang, P.-T., Mackem, S. \& Yang, Y. Indian hedgehog signals independently of PTHrP to promote chondrocyte hypertrophy. Development 135, 1947 LP - 1956 (2008).

22. Long, F. et al. Ihh signaling is directly required for the osteoblast lineage in the endochondral skeleton. Development 131, 1309 LP - 1318 (2004).

23. Lanske, B. et al. Ablation of the PTHrP gene or the PTH/PTHrP receptor gene leads to distinct abnormalities in bone development. The Journal of Clinical Investigation 104, 399-407 (1999).

24. Murakami, S. \& Noda, M. Expression of Indian Hedgehog During Fracture Healing in Adult Rat Femora. Calcified Tissue International 66, 272-276 (2000).

25. Vortkamp, A. et al. Regulation of Rate of Cartilage Differentiation by Indian Hedgehog and PTH-Related Protein. Science 273, 613 LP - 622 (1996).

26. Baht, G. S. et al. Exposure to a youthful circulaton rejuvenates bone repair through modulation of $\beta$ catenin. Nature Communications 6, 1-10 (2015).

27. Einhorn, T. A. The Wnt signaling pathway as a potential target for therapies to enhance bone repair. Science Translational Medicine vol. 2 42ps36-42ps36 (2010).

28. Wong, S. A. et al. $\beta$-catenin Signaling Regulates Cell Fate Decisions at the Transition Zone of the Chondro-Osseous Junction During Fracture Healing. bioRxiv 2020.03.11.986141 (2020) doi:10.1101/2020.03.11.986141. 
29. Bao, Q. et al. An appropriate Wnt/ß-catenin expression level during the remodeling phase is required for improved bone fracture healing in mice. Scientific Reports 7, 1-11 (2017).

30. Houben, A. et al. $\beta$-catenin activity in late hypertrophic chondrocytes locally orchestrates osteoblastogenesis and osteoclastogenesis. Development (Cambridge) 143, 3826-3838 (2016).

31. Li, Z. et al. Fracture repair requires TrkA signaling by skeletal sensory nerves. Journal of Clinical Investigation 129, 5137-5150 (2019).

32. Tomlinson, R. E. et al. NGF-TrkA Signaling by Sensory Nerves Coordinates the Vascularization and Ossification of Developing Endochondral Bone. Cell Reports 16, 2723-2735 (2016).

33. Tomlinson, R. E. et al. NGF-TrkA signaling in sensory nerves is required for skeletal adaptation to mechanical loads in mice. Proceedings of the National Academy of Sciences of the United States of America 114, E3632-E3641 (2017).

34. Rivera, K. O. et al. Local injections of $\beta-N G F$ accelerates endochondral fracture repair by promoting cartilage to bone conversion. Scientific Reports 10, 22241 (2020).

35. Langer, R. Drug delivery and targeting. Nature 392, 5-10 (1998).

36. Hoare, T. R. \& Kohane, D. S. Hydrogels in drug delivery: Progress and challenges. Polymer 49, 19932007 (2008).

37. D’souza, A. A. \& Shegokar, R. Polyethylene glycol (PEG): a versatile polymer for pharmaceutical applications. Expert Opinion on Drug Delivery vol. 13 1257-1275 (2016).

38. Choi, J. R., Yong, K. W., Choi, J. Y. \& Cowie, A. C. Recent advances in photo-crosslinkable hydrogels for biomedical applications. BioTechniques vol. 66 40-53 (2019).

39. Li, J. \& Mooney, D. J. Designing hydrogels for controlled drug delivery. Nature Reviews Materials vol. 1117 (2016).

40. Peña, J. R., Pinney, J. R., Ayala, P., Desai, T. A. \& Goldspink, P. H. Localized delivery of mechano-growth factor E-domain peptide via polymeric microstructures improves cardiac function following myocardial infarction. Biomaterials 46, 26-34 (2015).

41. Doroudian, G. et al. Sustained delivery of MGF peptide from microrods attracts stem cells and reduces apoptosis of myocytes. Biomedical Microdevices 16, 705-715 (2014). 
42. Finbloom, J. A., Demaree, B., Abate, A. R. \& Desai, T. A. Networks of High Aspect Ratio Particles to Direct Colloidal Assembly Dynamics and Cellular Interactions. Advanced Functional Materials 30, 1-9 (2020).

43. Champion, J. A. \& Mitragotri, S. Role of target geometry in phagocytosis. Proceedings of the National Academy of Sciences of the United States of America 103, 4930-4934 (2006).

44. Champion, J. A. \& Mitragotri, S. Shape induced inhibition of phagocytosis of polymer particles. Pharmaceutical Research 26, 244-249 (2009).

45. Ayala, P., Lopez, J. I. \& Desai, T. A. Microtopographical cues in 3D attenuate fibrotic phenotype and extracellular matrix deposition: implications for tissue regeneration. Tissue engineering. Part A 16, 25192527 (2010).

46. Ma, L. \& Zou, Y. TF-1 Cell Proliferation Assay Method for Estimating Bioactivity of Nerve Growth Factor. Journal of Applied Virology (2013) doi:10.21092/jav.v2i2.5.

47. Bahney, C. S. et al. Stem cell-derived endochondral cartilage stimulates bone healing by tissue transformation. Journal of Bone and Mineral Research (2014) doi:10.1002/jbmr.2148.

48. Cheng, Z. et al. Calcium-Sensing Receptors in Chondrocytes and Osteoblasts Are Required for Callus Maturation and Fracture Healing in Mice. Journal of Bone and Mineral Research (2020) doi:10.1002/jbmr.3864.

49. Hu, D. P. et al. Cartilage to bone transformation during fracture healing is coordinated by the invading vasculature and induction of the core pluripotency genes. Development (Cambridge) (2017) doi:10.1242/dev.130807.

50. Malhan, D. et al. An Optimized Approach to Perform Bone Histomorphometry. Frontiers in Endocrinology (2018) doi:10.3389/fendo.2018.00666.

51. Bahney, C. S. et al. Stem cell-derived endochondral cartilage stimulates bone healing by tissue transformation. Journal of Bone and Mineral Research (2014) doi:10.1002/jbmr.2148.

52. Shinoda, M. et al. Nerve growth factor contribution via transient receptor potential vanilloid 1 to ectopic orofacial pain. Journal of Neuroscience (2011) doi:10.1523/JNEUROSCI.0481-11.2011.

53. Hopkins, W. G. \& Slack, J. R. Effect of nerve growth factor on intramuscular axons of neonatal mice. Neuroscience (1984) doi:10.1016/0306-4522(84)90109-X. 
54. Sonnet, C. et al. Rapid healing of femoral defects in rats with low dose sustained BMP2 expression from PEGDA hydrogel microspheres. Journal of Orthopaedic Research 31, 1597-1604 (2013).

55. Stukel, J., Thompson, S., Simon, L. \& Willits, R. Polyethlyene glycol microgels to deliver bioactive nerve growth factor. Journal of Biomedical Materials Research Part A 103, 604-613 (2015).

56. Olabisi, R. M. et al. Hydrogel Microsphere Encapsulation of a Cell-Based Gene Therapy System Increases Cell Survival of Injected Cells, Transgene Expression, and Bone Volume in a Model of Heterotopic Ossification. Tissue Engineering Part A 16, 3727-3736 (2010).

57. Xu, L. et al. Molecular insights for the biological interactions between polyethylene glycol and cells. Biomaterials 147, 1-13 (2017).

58. Rot, C., Stern, T., Blecher, R., Friesem, B. \& Zelzer, E. A Mechanical Jack-like Mechanism Drives Spontaneous Fracture Healing in Neonatal Mice. Developmental Cell 31, 159-170 (2014).

59. Wang, Y., Newman, M. R. \& Benoit, D. S. W. Development of controlled drug delivery systems for bone fracture-targeted therapeutic delivery: A review. European Journal of Pharmaceutics and Biopharmaceutics (2018) doi:10.1016/j.ejpb.2018.02.023.

60. Ashley, G. W., Henise, J., Reid, R. \& Santi, D. V. Hydrogel drug delivery system with predictable and tunable drug release and degradation rates. Proceedings of the National Academy of Sciences 110, 2318 LP - 2323 (2013).

61. Henise, J., Hearn, B. R., Ashley, G. W. \& Santi, D. V. Biodegradable Tetra-PEG Hydrogels as Carriers for a Releasable Drug Delivery System. Bioconjugate Chemistry 26, 270-278 (2015).

62. Tria, M. A., Fusco, M., Vantini, G. \& Mariot, R. Pharmacokinetics of nerve growth factor (NGF) following different routes of administration to adult rats. Experimental neurology 127, 178-183 (1994).

63. Bertrand, A. A., Malapati, S. H., Yamaguchi, D. T. \& Lee, J. C. The Intersection of Mechanotransduction and Regenerative Osteogenic Materials. Advanced Healthcare Materials 9, (2020).

64. Song, S. et al. The synergistic effect of micro-topography and biochemical culture environment to promote angiogenesis and osteogenic differentiation of human mesenchymal stem cells. Acta Biomaterialia (2015) doi:10.1016/j.actbio.2015.02.021.

65. Parekh, S. H. et al. Modulus-driven differentiation of marrow stromal cells in 3D scaffolds that is independent of myosin-based cytoskeletal tension. Biomaterials 32, 2256-2264 (2011). 
66. Khatiwala, C. B., Kim, P. D., Peyton, S. R. \& Putnam, A. J. ECM compliance regulates osteogenesis by influencing MAPK signaling downstream of RhoA and ROCK. Journal of bone and mineral research : the official journal of the American Society for Bone and Mineral Research 24, 886-898 (2009).

67. Haffner-Luntzer, M. et al. Chronic psychosocial stress compromises the immune response and endochondral ossification during bone fracture healing via $\beta-A R$ signaling. Proc Natl Acad Sci USA 116, 8615 (2019).

68. Moncalvo, F., Martinez Espinoza, M. I. \& Cellesi, F. Nanosized Delivery Systems for Therapeutic Proteins: Clinically Validated Technologies and Advanced Development Strategies. Frontiers in Bioengineering and Biotechnology 8, 89 (2020).

69. Suk, J. S., Xu, Q., Kim, N., Hanes, J. \& Ensign, L. M. PEGylation as a strategy for improving nanoparticlebased drug and gene delivery. Advanced Drug Delivery Reviews 99, 28-51 (2016).

70. Bré, L. P., Zheng, Y., Pêgo, A. P. \& Wang, W. Taking tissue adhesives to the future: from traditional synthetic to new biomimetic approaches. Biomater. Sci. 1, 239-253 (2013). 